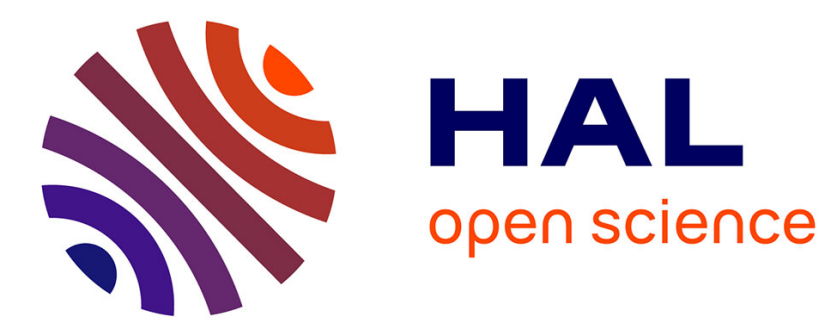

\title{
Abstention et blancs et nuls au référendum du 24 septembre 2000
}

Daniel Boy, Jean Chiche

\section{To cite this version:}

Daniel Boy, Jean Chiche. Abstention et blancs et nuls au référendum du 24 septembre 2000. Revue Française de Science Politique, 2001, 51 (1-2), pp.241 - 245. 10.3406/rfsp.2001.403617 . hal01016970v2

\section{HAL Id: hal-01016970 https://hal-sciencespo.archives-ouvertes.fr/hal-01016970v2}

Submitted on 3 Jul 2018

HAL is a multi-disciplinary open access archive for the deposit and dissemination of scientific research documents, whether they are published or not. The documents may come from teaching and research institutions in France or abroad, or from public or private research centers.
L'archive ouverte pluridisciplinaire HAL, est destinée au dépôt et à la diffusion de documents scientifiques de niveau recherche, publiés ou non, émanant des établissements d'enseignement et de recherche français ou étrangers, des laboratoires publics ou privés. 


\section{Abstention et blancs et nuls au référendum du 24 septembre 2000}

In: Revue française de science politique, 51e année, n¹-2, 2001. pp. 241-245.

Citer ce document / Cite this document :

Boy Daniel, Chiche Jean. Abstention et blancs et nuls au référendum du 24 septembre 2000. In: Revue française de science politique, 51e année, $n^{\circ} 1-2,2001$. pp. 241-245.

doi : 10.3406/rfsp.2001.403617

http://www.persee.fr/web/revues/home/prescript/article/rfsp_0035-2950_2001_num_51_1_403617 


\title{
ABSTENTION ET BLANCS ET NULS AU RÉFÉRENDUM DU 24 SEPTEMBRE $2000^{1}$
}

\author{
DANIEL BOY, JEAN CHICHE
}

$\mathrm{L}$ e référendum du 24 septembre sur la réduction du mandat présidentiel a fort peu mobilisé les électeurs : $69,8 \%$ d'entre eux se sont abstenus et $4,9 \%$ ont déposé un bulletin blanc ou nul (tableau 1). Les raisons de ce taux historique d'abstention sont connues : désintérêt pour une modification constitutionnelle que la plupart des Français semblaient prêts à accepter mais qui, du coup, paraissait acquise d'avance, polarisation vers d'autres enjeux politiques ou sociaux, qui rendait secondaire le vote au référendum.

Ce n'est pas seulement le taux d'abstention qui a atteint un sommet mais aussi la proportion de bulletins « blancs et nul $»^{2}: \mathrm{Si} 1^{\prime}$ 'on rapporte cette proportion aux seuls votants, on constate que $16,1 \%$ de ceux qui se sont déplacés jusqu'à un bureau de vote ne se sont exprimés ni.en faveur du oui ni en faveur du non. Même en admettant que parmi ces bulletins un certain nombre relèvent de l'erreur matérielle, il est clair que cette masse impressionnante de bulletins barrés ou surchargés, près de deux millions, a une signification politique sur laquelle il convient de s'interroger.

Tableau 1. Résultats France métropolitaine (chiffres ministère de l'Intérieur, France entière)

\begin{tabular}{l|c|c|c|c}
\hline & Nombre & \% aux inscrits & \% aux votants & \% aux exprimés \\
\hline Abstention & 27882504 & 69,8 & & \\
Blancs et nuls & 1940340 & 4,9 & 16,1 & \\
OUI & 7407697 & 18,5 & 61,4 & 73,2 \\
NON & 2710651 & 6,8 & 22,5 & 26,8 \\
Total & 39941192 & 100 & 100 & 100 \\
\hline
\end{tabular}

La comparaison de ces résultats avec quelques références historiques récentes portant sur des élections de toutes natures est éclairante (Tableau 2) :

Au précédent référendum, qui portait sur l'approbation du traité de Maastricht, moins du tiers des électeurs $(31,2 \%)$ avaient choisi l'abstention ou le bulletin blanc ou nul. Cette proportion avait atteint un premier record aux élections européennes de 1994 : $49,13 \%$. En revanche, le premier tour de l'élection présidentielle de 1995 avait été l'occasion d'une très forte mobilisation du corps électoral tandis que les législatives provoquées par la dissolution de 1997 n'avaient qu'assez médiocrement mobilisé les Français. Enfin, aux très récentes élections européennes de 1999, le pourcentage d'abstentionnistes et de blancs et nuls avait atteint un nouveau sommet.

1. Cette note de recherche reprend les éléments de base d'une communication à l'Association française de science politique le 27 septembre 2000. Tous les calculs sur lesquels reposent ce travail ont été effectués sur des fichiers électoraux recueillis au lendemain même du référendum au seul niveau géographique du département. Ces conclusions doivent donc être considérées comme une première esquisse. Elles devront être confirmées par des études plus fouillées exécutées à un niveau électoral plus fin (circonscription ou canton).

2. Sur l'évolution des blancs et nuls, cf., dans ce numéro, l'article d'Adélaïde Zulfikarpasic. 
Tableau 2. Abstention et blancs et nuls à cinq élections précédentes (chiffres métropole)

\begin{tabular}{l|c|c|c}
\hline & Abstention & Blancs et nuls & Abstention + Blancs et nuls \\
\hline Référendum 1992 & 28,9 & 2,4 & 31,2 \\
Européennes 1994 & 46,3 & 2,9 & 49,1 \\
Présidentielle 1995 & 20,5 & 2,2 & 22,7 \\
Législatives 1997 & 31,5 & 3,3 & 34,9 \\
Européennes 1999 & 52,2 & 2,8 & 55,1 \\
Référendum 2000 & 69,2 & 5 & 74,2 \\
\hline
\end{tabular}

En remontant plus avant dans l'histoire électorale française, on trouve d'autres exemples de référendums contestés : en 1972, le parti socialiste refusant de se prononcer sur le problème de l'entrée de la Grande-Bretagne dans le marché commun préconise l'abstention ou le vote blanc et, de fait, celui ci s'élève à 7,1\%. Dans le cas du référendum sur la réduction du mandat présidentiel il faut pourtant rappeler que seul le parti des chasseurs (Chasse pêche nature et tradition) donne une consigne de vote blanc.

Quelle signification accorder à l'abstention et au vote blanc et nul au référendum du 24 septembre 2000 ? Classiquement, on sait que ces deux phénomènes présentent des régularités de structures d'élection en élection. Une façon très élémentaire de le démontrer consiste à considérer les coefficients de corrélation de l'abstention et des blancs et nuls à la série d'élections mentionnées plus haut.

Tableau 3. Coefficients de corrélations

( $\mathrm{R}$ de Pearson) de l'abstention à six tours d'élections

\begin{tabular}{|l|c|c|c|c|c|}
\cline { 2 - 5 } \multicolumn{1}{c|}{} & $\begin{array}{c}\text { Référendum } \\
\mathbf{1 9 9 2}\end{array}$ & $\begin{array}{c}\text { Européennes } \\
\mathbf{1 9 9 4}\end{array}$ & $\begin{array}{c}\text { Présidentielle } \\
\mathbf{1 9 9 5}\end{array}$ & $\begin{array}{c}\text { Législatives } \\
\mathbf{1 9 9 7}\end{array}$ & $\begin{array}{c}\text { Européennes } \\
\mathbf{1 9 9 9}\end{array}$ \\
\hline Référendum 1992 & 1 & \multicolumn{2}{c}{} \\
\hline Européennes 1994 & 0,77 & 1 & \multicolumn{2}{c}{} \\
\hline Présidentielle 1995 & 0,84 & 0,67 & 1 & \multicolumn{1}{c}{} \\
\hline Législatives 1997 & 0,73 & 0,75 & 0,82 & 1 & 1 \\
\hline Européennes 1999 & 0,82 & 0,88 & 0,73 & 0,78 & 0,82 \\
\hline Référendum 2000 & 0,73 & 0,76 & 0,64 & 0,74 & \multicolumn{1}{c}{} \\
\hline
\end{tabular}

Tableau 4. Coefficients de corrélations

( $R$ de Pearson) des blancs et nuls à six tours d'élections

\begin{tabular}{|l|c|c|c|c|c|}
\cline { 2 - 5 } \multicolumn{1}{c|}{} & $\begin{array}{c}\text { Référendum } \\
\mathbf{1 9 9 2}\end{array}$ & $\begin{array}{c}\text { Européennes } \\
\mathbf{1 9 9 4}\end{array}$ & $\begin{array}{c}\text { Présidentielle } \\
\mathbf{1 9 9 5}\end{array}$ & $\begin{array}{c}\text { Législatives } \\
\mathbf{1 9 9 7}\end{array}$ & $\begin{array}{c}\text { Européennes } \\
\mathbf{1 9 9 9}\end{array}$ \\
\hline Référendum 1992 & 1 & \multicolumn{2}{c}{} \\
\hline Européennes 1994 & 0,84 & 1 & \multicolumn{2}{c}{} \\
\hline Présidentielle 1995 & 0,86 & 0,84 & 1 & 1 & \multicolumn{1}{c}{} \\
\hline Législatives 1997 & 0,87 & 0,84 & 0,86 & 0,86 & 1 \\
\hline Européennes1999 & 0,88 & 0,89 & 0,85 & 0,85 \\
\hline Référendum 2000 & 0,74 & 0,70 & 0,71 & 0,72 & 0,85 \\
\hline
\end{tabular}

On le constate dans les tableaux 3 et 4 , les coefficients de corrélations sont toujours très élevés même entre élections de natures très différentes.

Une autre manière de se représenter les régularités de distribution de ces phénomènes consiste à les cartographier. 
Carte 1. Abstention au référendum du 24 septembre 2000

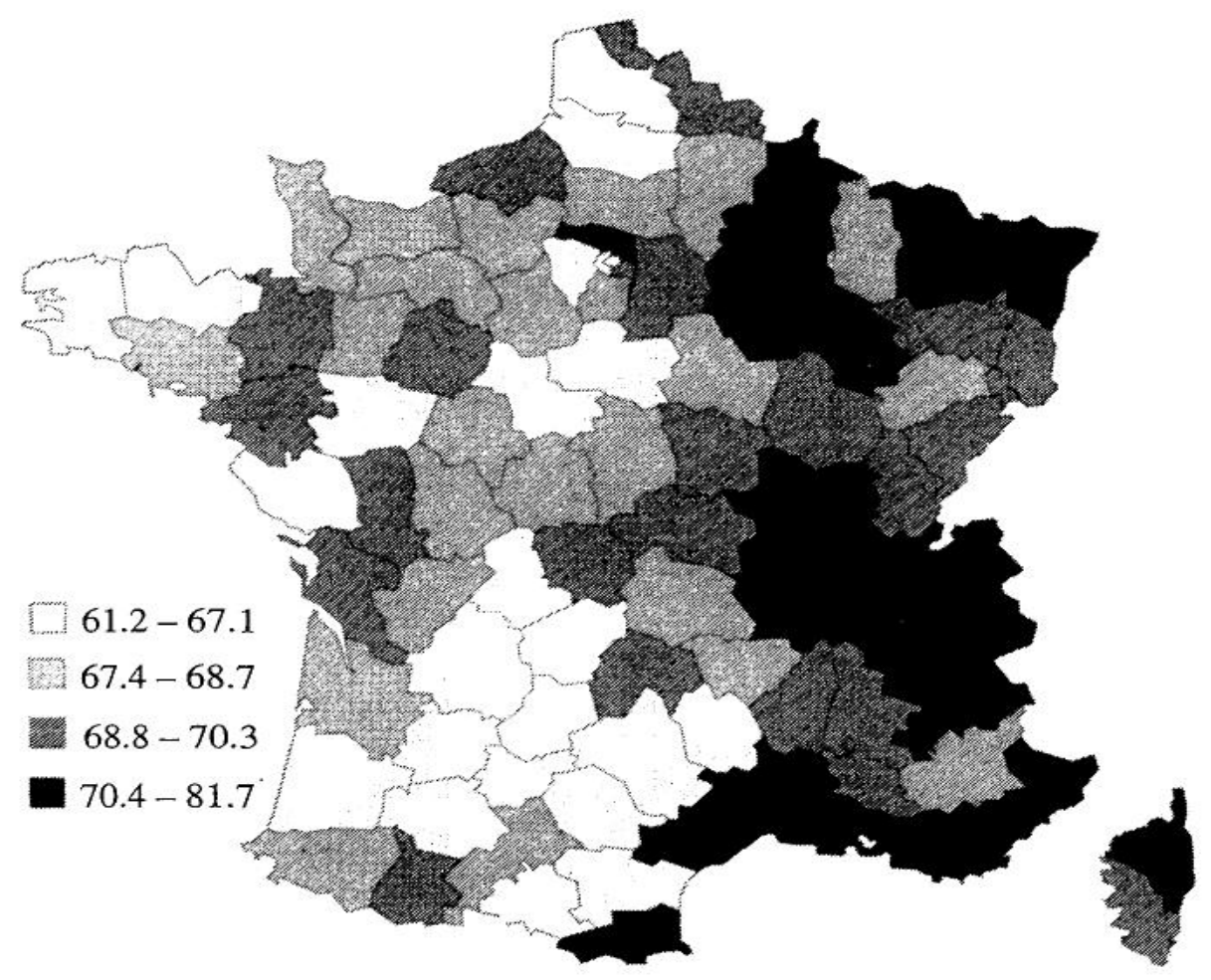

Carte 2. Blancs et nuls au référendum du 24 septembre 2000

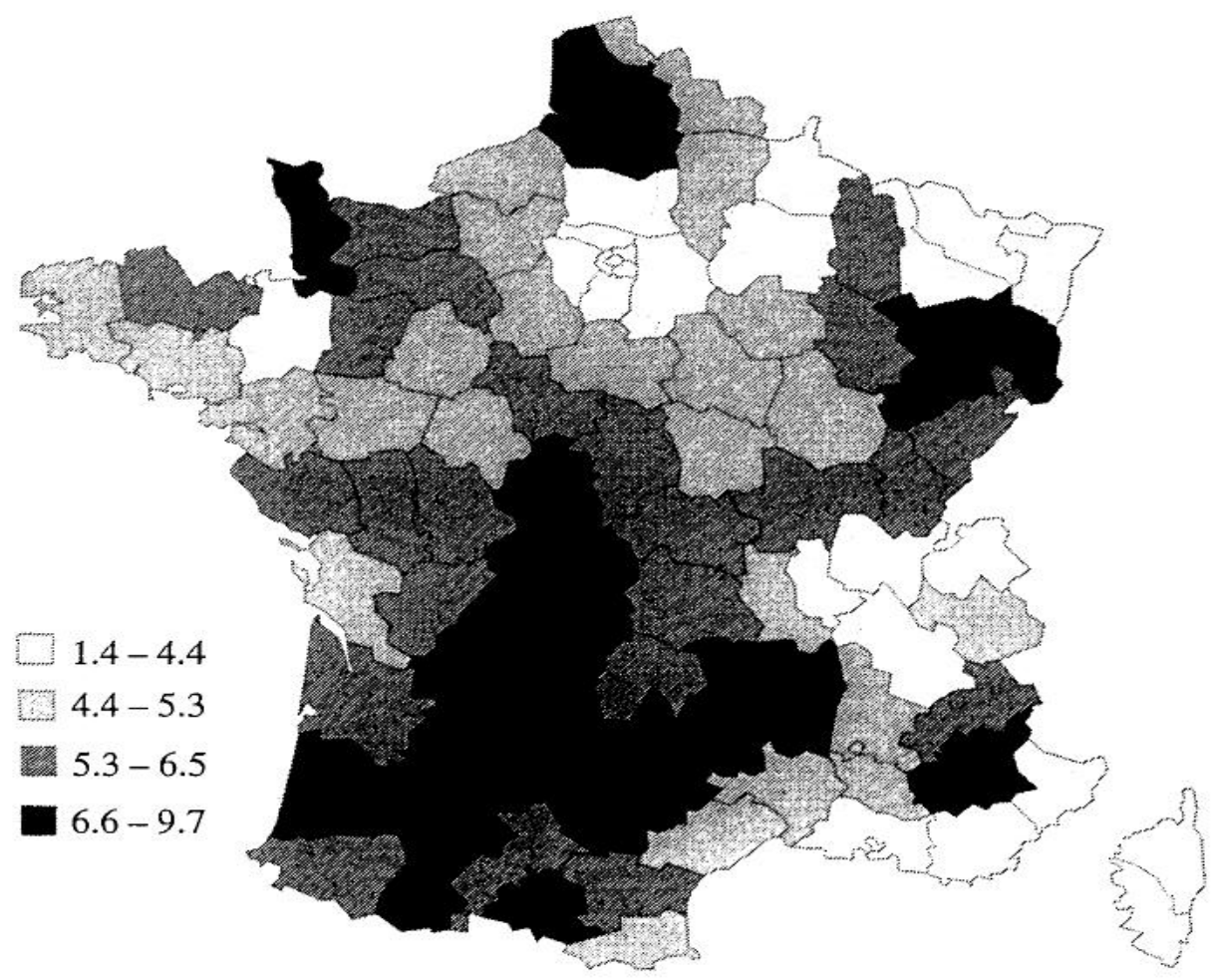


Les cartes 1 et 2 dessinent des structures géographiques assez familières aux électoralistes : l'abstention est fréquemment localisée dans l'est de la France de l'Alsace à la région Provence Côte d'Azur ; quant au blanc et nul, il dessine en bonne partie une carte de la ruralité. On sait aussi que les deux phénomènes que nous analysons ici, abstention et blanc et nul, entretiennent entre eux des relations contraires : il y a toujours un coefficient de corrélation négatif entre abstention et blanc et nul. Dans le cas du référendum du 24 septembre, il s'élève à -.70 .

Cette relation s'explique aisément: le niveau d'abstention est plus élevé en milieu urbain tandis que les blancs et nuls sont plus fréquents en milieu rural. On interprète cette différence en supposant que, dans les zones rurales le fait de se rendre ou non aux urnes le jour du vote est un acte socialement visible alors que, dans un contexte urbain, l'abstention demeure généralement anonyme. Là où l'électeur est sous le regard des autres le vote blanc permet de «s'abstenir » dans le secret de l'isoloir. tention.

Il y a donc en permanence un montant de vote blanc qui a, en réalité, valeur d'abs-

Mais on soupçonne aussi que, dans certaines circonstances, le fait de « voter blanc » a valeur d'opinion. Une offre politique incomplète entraîne souvent un réflexe protestataire de la fraction de l'électorat qui, le jour de l'élection, ne trouve pas « son candidat » ou «sa tendance ». Le phénomène est parfaitement visible quand les hasards du scrutin ont abouti à éliminer du second tour l'une des deux familles politiques majeures. Quand au second tour d'une élection législative ou cantonale ne subsistent que deux candidats de la même famille, gauche ou droite, le niveau d'abstention et de blancs et nuls devient considérable. Il en est de même, quoique de façon plus discrète, dans des circonstances où le second tour voit s'opposer un candidat de droite et un candidat de gauche, et où les électeurs d'extrême droite, suivant les consignes de leurs dirigeants, expriment leur rejet du «système ». Un exemple de cette situation : au second tour de l'élection présidentielle de 1995, le taux de blancs et nuls s'élève à $4,28 \%$ quand il n'était que de 2,21\% au premier tour. Or cette progression de 2,61 points de pourcentage est étroitement corrélée au vote pour Le Pen du premier tour (coefficient de corrélation : .89).

Qu'en est-il de ce vote blanc « protestataire » lors du référendum du 24 septembre?

Nous l'avons rappelé plus haut, le parti des chasseurs a officiellement appelé au vote blanc pour protester contre un référendum dont l'opportunité lui paraissait secondaire au regard d'autres problèmes. On connaît la structure géographique du vote en faveur de CPNT : elle est évidemment liée au caractère rural des unités géographiques et suit d'assez près le profil des régions de chasse traditionnelle : Sud-Ouest, Baie de Somme, etc. Aux élections européennes de 1999, le vote blanc s'élevait à 2,82 \%. Au référendum, il passe à $5 \%$. Or cette progression est assez fortement corrélée (.64) au niveau du vote en faveur de la liste menée par Saint Josse aux élections européennes de 1999.

Pour compléter ces résultats nous avons considéré les résultats obtenus dans un échantillon de 163 bureaux de vote représentatif de l'ensemble des bureaux de vote métropolitains et estimé la composition de l'abstention (et des blancs et nuls) à partir des résultats dans ces mêmes bureaux de vote aux élections européennes de 1999. On obtient une reconstitution mathématique (et théorique) des scores de l'abstention et des blancs et nuls qui donne la meilleure corrélation aux résultats réels. Les scores 
obtenus sont normés entre 0 et 1 , mais leur somme n'est pas normée. On interprétera donc ces résultats avec prudence. L'ordre de grandeur des taux obtenus reste néanmoins un précieux indicateur de ce qui a pu se passer ce 24 septembre 2000.

Concernant la participation, si l'on constate sans surprise que les abstentionnistes de 1999 ne se sont de nouveau pas déplacés pour aller voter, on remarquera que les électeurs communistes semblent avoir répondu au mot d'ordre de la direction de leur parti. Les électeurs d'extrême droite, frontistes ou mégretistes ont pour leur part semblé préférer l'abstention au vote NON.

Concernant les blancs et nuls, on notera enfin, parmi les listes de 1999, le taux le plus haut de la liste CPNT menée par Saint Josse.

Tableau 5. Estimations de la composition de l'abstention et des blancs et nuls aux listes des élections européennes de 1999 (sur un échantillon de 163 bureaux de vote)

\begin{tabular}{l|c|c}
\hline \multicolumn{1}{c|}{ Forces Européennes 1999 } & Abstention Référendum 2000 & $\begin{array}{c}\text { Blancs et nuls } \\
\text { Référendum 2000 }\end{array}$ \\
\hline ABS 99 & 0,97 & 0,01 \\
Blancs nuls & 0,21 & 0,66 \\
Laguiller & 0,64 & 0 \\
Hue & 0,88 & 0 \\
Hollande & 0,24 & 0,06 \\
Cohn-Bendit & 0,53 & 0,05 \\
Bayrou & 0,13 & 0,1 \\
Sarkozy & 0,31 & 0,07 \\
Pasqua & 0,51 & 0 \\
Le Pen & 0,66 & 0 \\
Mégret & 0,72 & 0 \\
Saint Josse & 0,49 & 0,34 \\
Divers & 0,76 & 0 \\
\hline
\end{tabular}

